MINI REVIEW

\title{
What is Already Known about Taste and Smell after Bariatric Surgery: A Mini-Review
}

\author{
Gabriel Pires dos Santos', Tassia Gomide Braga², and Luiz Guilherme Kraemer- \\ Aguiar $^{3^{*}}$
}

${ }^{1}$ Graduate Program in Medical Sciences. Faculty of Medical Sciences. State University of Rio de Janeiro (UERJ). Rio de Janeiro, Brazil

${ }^{2}$ Postgraduate Program in Clinical and Experimental Physiopathology (FISCLINEX). Faculty of Medical Sciences, State University of Rio de Janeiro, Rio de Janeiro, Brazil

${ }^{3}$ Obesity Unit, Policlínica Piquet Carneiro. Endocrinology, Department of Internal Medicine. Faculty of Medical Sciences, Biomedical Center, State University of Rio de Janeiro, Rio de Janeiro, Brazil

\begin{abstract}
Obesity is a public health problem that achieves pandemic proportions with important economic and social consequences, requiring aggressive intervention. From this, the different ways of treating this disease gain new dimensions.

A huge change in lifestyle in last decades, which includes a more sedentary life and a more access to tasty foods and calories, had imperative role in this scenario. Smell and taste seem to have a lot of influence on the choice of food. There are neuroendocrine pathways involved in these two senses.

Bariatric surgery has been shown to be effective in weight loss and control of comorbidities associated with obesity. In addition to changing the energy balance due to its restrictive and body mass and metabolic improvement.

We aimed to briefly review the literature regarding one aspect of the surgical treatment of obesity. Studies have shown that the surgical manipulation of the gastrointestinal tract could modify the secretions of gastric and intestinal peptides, and moreover, that these peptides are strictly related to hunger and satiety, and possibly to taste and smell. Some possible relationships with changes in taste, smell, and consequently appetite after bariatric surgery could occur, which would bring a new horizon to be studied in the field of obesity treatment.
\end{abstract} Open Access

Citation: dos Santos GP, Braga TG, Kraemer-AguiarLG. What is Already Known about Taste and Smell after Bariatric Surgery: A Mini-Review. Endocrinology Diabetes and Obesity. 2018; 1(2):4.

Received: July 30, 2018

Accepted: November 28, 2018

Published: January 14, 2019

Copyright: @ 2018 Kraemer-Aguiar LG. This is an open access article distributed under the terms of the Creative Commons Attribution License, which permits unrestricted use, distribution, and reproduction in any medium, provided the original author and source are credited.

\section{Corresponding author:}

Luiz Guilherme Kraemer-Aguiar,

Department of Internal Medicine.

Faculty of Medical Sciences, Biomedical

Center, State University of Rio de Janeiro,

Rio de Janeiro, Brazil

Email: Igkraemeraguiar@gmail.com.br

\section{Keywords}

Obesity, bariatric surgery, taste and smell

\section{Introduction}

Obesity is a chronic disease with a significant impact on global health, mainly due to the pandemic dimensions reached and also because of related comorbidities. Consequently, nowadays the prevalence is alarming. Diabetes, cardiovascular diseases, some types of cancer, sleep apnea and osteoarticular problems are some examples of clinical comorbidities closely related to this condition, which are responsible for increased morbimortality. ${ }^{1}$ Our communities have undergone profound changes in lifestyle, especially in handling and consuming of processed food products. 186,000 cases of death in 2011 in the United States of America were associated with an increase in body mass index (BMI). ${ }^{2}$ Latterly a large portion 
of the global population has access to abundant and varied food. The easier access to tasty and energy-dense foods contributed greatly to this epidemic. ${ }^{3}$ Besides that, the sensory appeal for high palatable foods (fat/sweet) seems to be reinforced in individuals with obesity and it leads to a perfect scenario for the increments in the prevalence of obesity. ${ }^{4}$

In our country, Brazil, data from Health Ministry indicated that over half of adult population is overweight $(54 \%)$ and that $18.9 \%$ have obesity, the latter being more prevalent in men. ${ }^{5}$ Recent epidemiological studies of 2015 show a global prevalence of $12 \%$ in adults, which amounts to 604 million obese adults in the world. ${ }^{6}$

The cephalic phase is the anticipatory physiological regulation related to feeding. The food smell has an important role in food palatability and intake. ${ }^{7}$ This effect could be mediated by neural pathways and interfere on quantity and quality of calories ingested. The sense of taste is recognized to be a driving to food choice. During the prandial period, the sense of taste plays a major role in the decision to consume or to avoid foods. ${ }^{8}$

Recent studies have suggested that the endocrine system modulates smell and taste capacity. Some gut hormones like ghrelin, cholecystokinin (CCK), peptide YY (PYY) and glucagon-like peptide-1 (GLP-1) have been linked to the smell-taste capacity. ${ }^{9}$

This mini review aims to discourse about the role of bariatric surgery on obesity treatment, with especial phocus on the possible secondary changes on taste and smell possibly through mechanisms that involves gut hormones.

\section{Materials and Methods}

A search of the PubMed database was perfomed for studies with relevance to the topic obesity, bariatric surgery and changes in taste and smell. The terms "taste", "smell", "bariatric surgery", "obesity treatment" and "weight regain" were used for this search. We have also added an ongoing study developed by our group entitled "Changes in Taste After Bariatric Surgery as a Factor to Weight Loss Maintenance" and presented at the Obesity Week 2017 in Washington DC, U.S.A.

\section{Pathophysiology of Obesity}

Obesity is a neurometabolic disease with some particular sites of defects, mainly at adipose tissue, digestive system, and central nervous system. Concerning adipose tissue, one of the most studied hormones in the genesis of obesity is leptin. This peptidic hormone produced in adipose tissue acts in the hypothalamus regulating food intake, inhibiting orexigenic pathways and stimulating satiety. ${ }^{10}$ The vast majority of subjects with obesity present elevated levels of leptin, but a dysfunctional signaling pathway in the hypothalamus that leads to resistance to leptin's action. ${ }^{11}$ The digestive system is involved in the production and secretion of some gastrointestinal peptides, including glucose-dependent insulinotropic polypeptide (GIP), GLP-1 and PYY.12 These hormones are secreted when food get in touch with the small intestine. They rise up to two hours after a meal, while at fasting very low basal levels are present.

One of the treatment options for obesity is a type of bariatric surgery that bypasses the upper portion of the small intestine and promotes a rapid forthcoming of food to the small intestine (named as Roux-en-Y gastric bypass). This invasive procedure lead to a considerable increase in the levels of intestinal peptides. ${ }^{13}$ Since these peptides act as satietogenic agents in the central nervous system, not only the gastric restriction promoted by surgery but also these peptides are responsible for weight loss. ${ }^{13}$

Analogs for GLP-1 are on the market and have shown beneficial effects not only on obesity but also on diabetes. ${ }^{12}$ For this reason, other peptides, with possibly similar benefits, are candidates for the treatment of obesity. Ghrelin and CKK are participants of similar processes. The first is produced mainly in the gastric mucosa and acts on the hypothalamus as a hunger stimulant while CKK is produced mainly in the duodenum and jejunum in response to protein and fat intake. ${ }^{13}$

The interesting point is that there is also an action of intestinal hormones on taste. CKK and neuropeptide $\mathrm{Y}$ (NPY) were the first hormones described in rat taste cells. CKK receptors in gustative buds of the tongue are involved in the sweet taste response, including artificial sweeteners. ${ }^{14}$ Taste cells on tongue produces hormones, such as GLP-1 and ghrelin. ${ }^{15}$ Also, afferent nerve fibers in gustative buds contain receptors for locally produced hormones such 
as GLP-1 and NPY, that are also able to modulate taste signal.

In respect of taste, the function of NPY is antagonistic to that of CKK causing a decrease in the response of the sweet signals and also of the umami taste. This signaling amplifies the perception of bitter. ${ }^{16}$ The PYY secreted in the intestinal L-cells is also produced by the taste cells. Knockout mice for PYY receptors showed a considerable reduction in preference for fat in taste. ${ }^{17}$ Rats deficient in GLP-1 receptors had a dramatic reduction in responses of sucrose and sucralose taste (artificial sweeteners) and increased sensitivity to umami taste. ${ }^{18}$ Glucagon, produced by pancreatic $\alpha$-cells, also has receptors on taste cells. Its genetic or pharmacological absence decreases the response to sweet taste while ghrelin, secreted bystomach, has receptors on all types of taste cells. ${ }^{19-21}$ Knockout mice forghrelin receptors have a considerable reduction in salty and sour taste. ${ }^{21}$ In this sense, the hormonal signals derived from intestinal peptides influence not only hunger and saciety but also food taste and possible food preferences, and may be associated with obesity.

\section{Bariatric surgery and the new horizons for the treatment of obesity}

Nowadays, some of options offered for obesity are: changes in lifestyle behaviors (dieting and exercise), pharmacotherapy and bariatric surgery. The first is the less invasive therapy in the treatment of obesity and when combined with pharmacotherapy or surgery brings better results. Although lifestyle modifications should be present for all options of treatment, surgery is indicated to: patients between 18 and 60 years old with BMI $\geq 40 \mathrm{~kg} / \mathrm{m}^{2}$ or BMI $35-40 \mathrm{~kg} / \mathrm{m}^{2}$ associated with comorbidities in which surgically induced weight loss is expected to improve them (i.e., arterial hypertension and diabetes, sleep apnea, non-alcoholic steatosis of the liver, among others). Particular cases related to obesity in children and teenagers and also in individuals older than 60 years should be discussed by a multi-professional team considering risk- and also cost-benefits aspects. ${ }^{22}$

Bariatric surgery is the most invasive treatment offered for obesity treatment. Although, it is the most effective one. This therapy leads to a considerable loss of weight, especially in the first two years after surgery. The beneficial effects of surgery are not limited to loss of weight but also to amelioration or even remission of many obesity-related comorbidities. Studies showed a considerable improvement in the health of the individual after bariatric surgery, especially regarding the remission of type 2 diabetes and reduction of factors that compose the metabolic syndrome (i.e., hyperglycemia, hypertrygliceridemia, low HDL-cholesterol, hypertension and higher levels of waist circumference). ${ }^{23,24}$ The mortality rate in the procedure is meager when performed by a trained professional, getting nowadays close to $0.35 \%{ }^{25}$ These facts, associated with lower total morbimortality after bariatric surgery, promoted increased rates of bariatric procedures worldwide.

Bariatric surgery acts aggressively reducing caloric intake which occurs not only due to anatomical restriction and disabsorption but also because of alterations in the secretion of gastrointestinal anorexigenic and orexigenic substances. This procedur not only reduces the appetite but also promotes a state of precocious saciety. Another important aspect involved in bariatric surgery effects and not yet studied entirely are the possible changes gustatory system secondary to changes in intestinal peptides. Recent studies report that there is an essential alteration in taste, smell and post-surgery food preferences and these altered responses would supposedly help patients maintain long-term sustained weight loss, acting as an adjuvant effect on appetite. ${ }^{26,27}$ Such changes may be present to any of bariatric techniques currently used. However, studies indicate that taste, smell and food-preferences changes are more noticeable in the roux-en-Y gastric bypass technique.

\section{Changes in taste and smell after bariatric surgery}

As we have mentioned above, sensory responses to the taste, smell, and texture of foods help determine food preferences and eating behaviors.

Through taste, our organism comes in contact with substances which will bring various sensations, good or bad ones. The tongue is the main component of the gustatory system which is composed of taste buds and papillae both sited on gustatory buttons. Once in contact with the tongue, the food is decoded, texture, temperature, and taste are perceived. Taste has five classes: salty, sweet, sour, bitter and umami and depending on the composition of the food, sodium channels, hydrogen, glutamate, among others; can be activated. This activation generates a signal which is transmitted to the central nervous system and decoded as one of these classes of taste mentioned above. Since bariatric surgeries can induce changes in the 
secretion of the incretins, especially GLP-1 increments, and that this hormone has known receptors, not only in the hypothalamus but also on the tongue, and on the taste buds, it is expected that an altered taste perception may occur after these surgeries. ${ }^{28}$ Given all data mentioned above, the modification of the taste in foods after a bariatric procedure, especially those that promote an altered intestinal transit may have some influence in long-term weight maintenance and should be investigated as a positive predictor for lower rates of regaining weight. On this view, a study investigated patients who have undergone Roux-en-Y gastric bypass and showed reduced proportion of calorie intake from sweets and high-calorie foods. ${ }^{29}$ Another study observed a changing perception for the palatability of sweets from pleasurable to non-pleasurable one after Roux-en-Y gastric bypass. ${ }^{30}$

Concerning smell, it is known that it is one of the less developed senses in humans when compared to other animals and there is also a tremendous subjective appeal to the sensations generated by the smell. It is notorious the effects produced by the smell of pleasant food to stimulate not only the onset of processes such as salivation and stomach acid production but also the desire to eat and hunger. One aspect in those individuals subjected to bariatric surgery occurred early after surgery, a sense of modified olfaction for some foods, especially in the initial months post surgery which paralleled to the period of more significant weight loss, returning to normal in most cases in approximately six months. ${ }^{30}$ Although bias involving psychological issues may have occured influencing olfaction, such results suggest that bariatric surgery can also act on the olfactory centers of the nervous system.

To evaluate the changes after bariatric surgery Graham and co-workers applied a questionnaire in 188 patients submitted to Roux-en-Y gastric bypass investigating not only the expected changes in appetite but also possible alterations in the sense of smell and taste, and additionally food aversion. They observed marked changes in appetite, taste, and smell in $97 \%, 73 \%$ and $42 \%$ of patients, respectively. ${ }^{31}$ We also decided to investigate it and additionally to associate these possible changes with weight regain after bariatric surgery. Forty-two patients subjected to bariatric surgery followed at the outpatient's clinic of the State University of Rio de Janeiro answered a questionnaire created by Tichansky and co-workers and modified by Graham and co-workers. ${ }^{31,32}$ We divided groups according to levels of weight regain in less than $10 \%$ and more than it. ${ }^{32,33}$ Those who had less weight regain showed more changes in the taste of the food suggesting that perception of a taste of the foods were associated with changes in weight after bariatric surgery. We are still recruiting and testing additional volunteers but, in our population, this data confirms the idea that changes in taste perception could have an influence on weight regain after bariatric surgery, and possibly, should be viewed as a protective factor against weight regain.

\section{Conclusion}

As already known bariatric surgery not only promotes anatomical changes but also brings about changes on peptides secreted by the gastrointestinal tract. There is a gap of knowledge about how these changes, related to nervous and gustative system pathways can affect weight loss and also estimulate weight maintenance after the procedure. Some studies, based on questionnaires, have shown a modification in taste and smell perceptions after bariatric surgery. The relationship between these changes in taste and smell with the postoperative weight maintenance and regain needs further elucidation but could be viewed as a possible predictive protective factor against weight regain. The role of gut hormones on these observations has also yet to be proved. Aspects relating taste and smell and their pathophysiology need to be studied and bariatric surgery should be viewed as a model and may add to knowledge to this. Further studies are needed to better define if post bariatric changes in taste and smell occur and also if they could be viewed as predictive factors involved on weight changes. If so, inferences about possible the use of drugs directed to smell and taste can be thought for the future in the treatment of obesity.

\section{Funding information}

The National Council for Scientific and Technologic Development (CNPq) and the Carlos Chagas Filho Foundation for Research Support in the State of Rio de Janeiro (FAPERJ) supported this work by scholarships for all authors. 


\section{Author's contribution}

GPS searched data and wrote the paper. TGB revised the paper. LGK-A designed the manuscritpt, made critical revision and revised the paper.

\section{Conflict of interest}

The authors declared no conflict of interest.

\section{References}

1. Andolfi C, Fisichella P. Epidemiology of Obesity and Associated Comorbidities. J Laparoendosc Adv Surg Tech. 2018;28(8): 919-924. doi:10.1089/lap.2018.0380

2. Preston S, Vierboom Y, Stokes A. The role of obesity in exceptionally slow US mortality improvement. Proc Natl Acad Sci. 2018;115(5): 957-961. doi:10.1073/pnas.1716802115

3. Besnard P. Lipids and obesity: Also a matter of taste? Rev Endocr Metab Disord. 2016;17(2):159-170. doi:10.1007/s11154-016-9355-2

4. Drewnowski A, Brunzell J, Sande K, Iverius P, Greenwood M. Sweet tooth reconsidered: Taste responsiveness in human obesity. Physiol Behav. 1985;35(4):617-622. doi:10.1016/00319384(85)90150-7

5. Health Ministry. Vigitel Brazil 2017: surveillance of risk and protective factors for chronic diseases by telephone survey: estimates of sociodemographic frequency and distribution of risk and protective factors for chronic diseases in the capitals of the 26 Brazilian states and the Federal District in 2017. Brasilia: Health Ministry. 2018: 35-45.

6. Matta J, Carette C, Rives Lange C, Czernichow S. Épidémiologie de l'obésité en France et dans le monde. La Presse Médicale. 2018;47(5): 434-438. doi:10.1016/j.Ipm.2018.03.023

7. Massolt E, van Haard P, Rehfeld J, et al. Appetite suppression through smelling of dark chocolate correlates with changes in ghrelin in young women. Regul Pept. 2010;161(1-3):81-86. doi: 10.1016/j.regpep.2010.01.005

8. Besnard P. Lipids and obesity: Also a matter of taste?.Rev Endocr Metab Disord.2016;17(2):159-170. doi:10.1007/s11154-016-9355-2

9. Berthoud H, Morrison C. The Brain, Appetite, and Obesity. Annu Rev Psychol. 2008;59(1): 55-92. doi:10.1146/annurev.psych.59.103006.093551

10. Park H, Ahima R. Physiology of leptin: energy homeostasis, neuroendocrine function and metabolism. Metabolism. 2015;64(1): 24-34. doi:10.1016/j.metabol.2014.08.004

11. Kelesidis T. Narrative Review: The Role of Leptin in Human Physiology: Emerging Clinical Applications. Ann Intern Med. 2010;152(2):93. doi:10.7326/0003-4819-152-2-201001190-00008

12. Adamska E, Ostrowska L, Górska M, Krętowski A. The role of gastrointestinal hormones in the pathogenesis of obesity and type 2 diabetes. Gastroenterology Rev. 2014;2: 69-76. doi: 10.5114/pg.2014.42498

13. Dimitriadis G, Randeva M, Miras A. Potential Hormone Mechanisms of Bariatric Surgery. Curr Obes Rep. 2017;6(3): 253-265. doi:10.1007/s13679-017-0276-5

14. Zhao F-L, Shen T, Kaya N, et al. Expression, physiological action, and coexpression patterns of neuropeptide Y in rat taste-bud cells. Proc Natl Acad Sci. 2005;102(31): 11100-11105. doi:10.1073/pnas.0501988102.

15. Shin Y, Martin B, Golden E, et al. Modulation of taste sensitivity by GLP-1 signaling. J Neurochem. 2008;106(1): 455-463. doi:10.1111/j.1471-4159.2008.05397.x

16. Herness S, Zhao F. The neuropeptides CCK and NPY and the changing view of cell-to-cell communication in the taste bud. Physiol Behav. 2009;97(5): 581-591. doi: 10. 1016/ j.physbeh. 2009.02.043

17. Martin B, Dotson C, Shin Y, et al. Modulation of Taste Sensitivity by GLP-1 Signaling in Taste Buds. Ann N YAcad Sci. 2009;1170(1):98-101. doi:10.1111/j.1749-6632.2009.03920.x

18. Elson A, Dotson C, Egan J, Munger S. Glucagon signaling modulates sweet taste responsiveness. The FASEB J. 2010;24(10): 3960-3969. doi:10.1096/fj.10-158105 
19. La Sala M, Hurtado M, Brown A, et al. Modulation of taste responsiveness by the satiation hormone peptide YY. The FASEB J. 2013;27(12): 5022-5033. doi:10.1096/fj.13-228064

20. Shin Y, Martin B, Kim W, et al. Ghrelin Is Produced in Taste Cells and Ghrelin Receptor Null Mice Show Reduced Taste Responsivity to Salty (NaCl) and Sour (Citric Acid) Tastants. PLoS ONE. 2010;5(9) :e12729. doi:10.1371/journal.pone.0012729

21. Kang K, Zmuda E, Sleeman M. Physiological role of ghrelin as revealed by the ghrelin and GOAT knockout mice. Peptides. 2011;32(11): 2236-2241. doi:10.1016/j.peptides.2011.04.028

22. Fried M, Yumuk V, Oppert J,et al. Interdisciplinary European Guidelines on Metabolic and Bariatric Surgery. Obesity Surgery, 2013;24(1): 42-55.

23. Buchwald H, Avidor Y, Braunwald E, et al. Bariatric Surgery. JAMA. 2004;292(14):1724. doi:10.1001/jama.292.14.1724

24. Gloy V, Briel M, Bhatt D, et al. Bariatric surgery versus non-surgical treatment for obesity: a systematic review and meta-analysis of randomised controlled trials. BMJ. 2013;347(1): f5934-f5934. doi:10.1136/bmj.f5934

25. Buchwald H, Estok R, Fahrbach K, Banel D, Sledge I. Trends in mortality in bariatric surgery: a systematic review and meta-analysis. Surgery. 2007;142(4): 621-632.

26. Holinski F, Menenakos C, Haber G, Olze H, Ordemann J. Olfactory and Gustatory Function After Bariatric Surgery. Obesity Surgery. 2015;25(12): 2314-2320. doi:10.1007/s11695-015-1683-x.

27. Behary P, Miras AD. Food preferences and underlying mechanisms after bariatric surgery. Proc Nat Acad Sci.2015;74(4): 419-425. doi:10.1017/s0029665115002074

28. Laferrère B. Bariatric surgery and obesity: influence on the incretins. Int J Obes Suppl. 2016;6(S1). doi:10.1038/ijosup.2016.8.

29. Olbers T, Björkman S, Lindroos A, et al. Body Composition, Dietary Intake, and Energy Expenditure After Laparoscopic Roux-en-Y Gastric Bypass and Laparoscopic Vertical Banded Gastroplasty. Ann Surg. 2006;244(5):715-722. doi:10.1097/01.sla.0000218085.25902.f8

30. Pepino MY, Bradley D, Eagon JC, et al. Changes in taste perception and eating behavior after bariatric surgery-induced weight loss in women. Obesity. 2013;22(5). doi:10.1002/oby.20649.

31. Graham L, Murty G, Bowrey DJ. Taste, smell and appetite change after Roux-en-Y gastric bypass surgery. Obes Surg. 2014; 24(9): 1463-1468. doi: 10.1007 /s11695-014-1221-2.

32. Tichansky DS, Boughter JD, Madan AK. Taste change after laparoscopic Roux-en-Y gastric bypass and laparoscopic adjustable gastric banding. Surg Obes Relat Dis.2006;2(4): 440-444. doi:10. 1016/j.soard.2006.02.014.

33. Santos GP, Braga TG, Kraemer-Aguiar LG. Changes in Taste After the Bariatric Surgery as a Factor to Weight Loss Maintenance. Poster presented at: OBESITY WEEK 2017, Washington, DC. 